\title{
A new role for host annexin $A 2$ in establishing bacterial adhesion to vascular endothelial cells: lines of evidence from atomic force microscopy and an in vivo study
}

\author{
Xi He ${ }^{1} \cdot$ Weiwei Zhang ${ }^{2,3} \cdot$ Qing Chang ${ }^{1} \cdot$ Zhengchen Su$^{1} \cdot$ Dejun Gong ${ }^{2} \cdot$ Yixuan Zhou $^{2} \cdot$ Jie Xiao $^{1}$ •

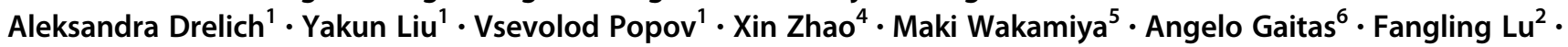 \\ Bin Gong ${ }^{1}$
}

Received: 16 March 2019 / Revised: 8 May 2019 / Accepted: 20 May 2019 / Published online: 28 June 2019

(c) The Author(s), under exclusive licence to United States and Canadian Academy of Pathology 2019

\begin{abstract}
Understanding bacterial adhesion is challenging and critical to our understanding of the initial stages of the pathogenesis of endovascular bacterial infections. The vascular endothelial cell (EC) is the main target of Rickettsia, an obligately intracellular bacterium that causes serious systemic disease in humans and animals. But the mechanism(s) underlying bacterial adherence to ECs under shear stress from flowing blood prior to activation are unknown for any bacteria. Although host surface annexin a2 (ANXA2) has been identified to participate in efficient bacterial invasion of epithelial cells, direct evidence is lacking in the field of bacterial infections of ECs. In the present study, we employ a novel, anatomically based, in vivo quantitative bacterial-adhesion-to-vascular-EC system, combined with atomic force microscopy (AFM), to examine the role of endothelial luminal surface ANXA2 during rickettsial adherence to ECs. We also examined whether ANXA2 antibody affected binding of Staphylococcus aureus to ECs. We found that deletion of ANXA2 impeded rickettsial attachment to the ECs in vitro and blocked rickettsial adherence to the blood vessel luminal surface in vivo. The AFM studies established that EC surface ANXA2 acts as an adherence receptor for rickettsiae, and that rickettsial adhesin OmpB is the associated bacterial ligand. Furthermore, pretreatment of ECs with anti-ANXA2 antibody reduced EC surface-associated S. aureus. We conclude that the endothelial surface ANXA2 plays an important role in initiating pathogen-host interactions, ultimately leading to bacterial anchoring on the vascular luminal surface.
\end{abstract}

These authors contributed equally: Xi He, Weiwei Zhang

Supplementary information The online version of this article (https:// doi.org/10.1038/s41374-019-0284-z) contains supplementary material, which is available to authorized users.

$\triangle$ Fangling Lu

lufanglin@smmu.edu.cn

$\bowtie$ Bin Gong

bigong@utmb.edu

1 Department of Pathology, University of Texas Medical Branch, Galveston, TX 77555, USA

2 Department of Cardiovascular Surgery, Changhai Hospital, 200433 Shanghai, China

\section{Introduction}

The ability of invasive bacteria to adhere to host cell surfaces is a pivotal initial step in successfully establishing infection of an obligate or facultative intracellular parasitic bacterium [1-10]. In bloodstream infections, for most bacterial microorganisms that are generally not considered intracellular pathogens, the establishment of metastatic endovascular infections after adhering to vascular

3 Department of Laboratory Diagnostics, Changhai Hospital, 200433 Shanghai, China

4 Department of Animal Science, McGill University, 21111 Lakeshore Road, Ste. Anne de Bellevue, QC, Canada

5 Department of Neurology, University of Texas Medical Branch, Galveston, TX 77555, USA

6 The Estelle and Daniel Maggin Department of Neurology, Icahn School of Medicine at Mount Sinai, 1468 Madison Ave, New York, NY 10029, USA 
endothelial cells (ECs) is one of the major determinants in disease outcome [11-17]. Understanding how bacteria attach to host cells is a crucial challenge in microbiology, and in medicine is a key for controlling bacterial infections at early stages [18]. Remarkably, in animal models mimicking sepsis, several Staphylococcus aureus proteins have been shown to mediate the adhesion of $S$. aureus to activated ECs that were stimulated by an initial infection with a low dose of bacteria $24 \mathrm{~h}$ before reinfection with a higher dose $[11,12,19]$. However, the mechanism(s) underlying bacterial adherence to ECs under shear stress from flowing blood prior to activation are completely unknown. This knowledge is critical to our understanding of the initial stages of the pathogenesis of endovascular bacterial infections, which is needed for developing therapeutics that could limit infection at this initial stage.

Obligately intracellular bacteria in the genus Rickettsia $(R$.) are the causative agents of spotted fever group (SFG) rickettsioses [20-22], including $R$. rickettsii [22, 23], $R$. conorii [24], and $R$. australis [25, 26]. Typically, rickettsiae are transmitted through the bite of infected ticks, and ECs are the primary vertebrate host target cells $[11,27]$. The initial step in establishing a productive intracellular infection is for the bacterium to recognize and establish an adhesive interaction with specific cellular receptor(s) to firmly anchor itself on the host EC luminal surface, thus overcoming detachment by shear stress from blood flow prior to invasion into the EC [11, 28]. Therefore, rickettsial infection is a suitable model to employ for studying endovascular bacterial adhesion. Remarkable insights into the rickettsial components involved in this initial interaction have come from identification of rickettsial adhesins [29-35], although comparatively little is known about host surface receptor(s) and the mechanism for establishing the connection between the host cell surface and rickettsiae. Host proteins $\mathrm{Ku} 70$ [36], $\alpha 2 \beta 1$ integrin [29], clathrin [37], caveolin 2 [37], and exchange protein activated by cAMP (EPAC) [38] have been identified as being involved in rickettsial invasion into nonphagocytic host cells via endocytic mechanisms [36]. Yet, as analyzed by an immunofluorescence (IF)-based assay, only the $\alpha 2 \beta 1$ integrin heterodimer [29] and EPAC1 [38] were shown to be involved in rickettsial adhesion to the host cell surface. $\alpha 2 \beta 1$ integrin mainly serves as a endothelial receptor for extracellular matrix molecules [39]. EPAC is an intracellular cAMP receptor [40] and is speculated to play a regulatory role, rather than as a direct receptor for rickettsial adherence on the host cell surface. Using a functional antibody specific to KU70, it was shown that rickettsial invasion into Vero cells was effectively blocked, but there was no effect on rickettsial adhesion to Vero cell surfaces [36].

ECs express abundant plasminogen (Plg), and Plg activator binding sites on their vascular luminal surfaces serve plasmin-based fibrinolytic functions [41], among which the annexin A2 (ANXA2) is the best recognized and is emerging as the focus of research on a growing spectrum of biologic and pathologic processes [42, 43]. ANXA2 is a $\mathrm{Ca}^{2+}$-regulated and phospholipid-binding protein that associates with cell membrane lipid rafts and the actin cytoskeleton [42, 44, 45]. It is detected on endothelial surfaces in the form of a complex with S100A10, (ANXA2$\mathrm{S} 100 \mathrm{~A} 10)_{2}$. Of note, there is in vitro evidence that ANXA2 participates in efficient invasion of S. aureus [46], Escherichia coli [47, 48], Pseudomonas aeruginosa [49], Salmonella typhimurium [50], and Mycoplasma pneumoniae [51] in epithelial cells via regulation of cytoskelton remodeling in the vicinity of lipid rafts. Neutralization of the ligands on $P$. aeruginosa by incubation of the bacteria with recombinant, soluble ANXA2 prevents bacterial entry into human epithelial cells, suggesting ANXA2 may be a receptor for bacterial adherence and/or invasion [49]. Although ANXA2 has been identified as a binding partner of $S$. aureus adhesin clumping factor A in a protein-protein binding assay [46], direct in vitro or in vivo evidence are completely lacking in the field of endovascular infections after adherence to ECs.

In the present study, we employ a novel, anatomically based, in vivo quantitative bacterial-adhesion-to-vascular$\mathrm{EC}$ analysis system, combined with atomic force microscopy (AFM), to examine the role of endothelial luminal surface ANXA2 during rickettsial adherence to ECs. We identified endothelial surface ANXA2 as a receptor for SFG rickettsial adhesion in vivo using AFM probes coated with recombinant $R$. conorii adhesin OmpB (reOmpB) to assess dynamic interactions during rickettsial adhesion. Our results demonstrated that recombinant ANXA2 (reANXA2), compared to bovine serum albumin (BSA) and Plg, has the most binding ability to reOmpB during measurement of protein-protein interaction forces. We also used reOmpBcoated probes with single living human umbilical vein endothelial cells (HUVECs) and demonstrated that reOmpB exerted an ANXA2-dependent binding strength to the living endothelial surface. Moreover, an ANXA2 monoclonal antibody could attenuate adhesion of $S$. aureus, a versatile facultative intracellular pathogen, to human ECs.

\section{Materials and methods}

\section{Mice}

All animal experiments were performed according to protocols approved by the Institutional Animal Care and Use Committee of the University of Texas Medical Branch (UTMB). Wild-type (WT) mice (C57BL/6J) were obtained from Jackson Laboratory (Bar Harbor, ME). Anxa2-null mice, on the $\mathrm{C} 57 \mathrm{BL} / 6 \mathrm{~J}$ background, were a generous gift 
from Dr Katherine Hajjar (Weill Cornell Medicine, New York, NY) [52]. All mice used in this study were 8 to 12week-old males. C57BL/6J mice are highly susceptible to $R$. australis. Therefore, this organism was chosen as the SFG rickettsial agent of choice [25].

\section{Rickettsiae and S. aureus}

R. australis (strain Cutlack) was prepared as described [38]. Uninfected Vero cells were processed as mock control material using the same procedure. All biosafety level (BSL) 3 or ABSL3 experiments were performed in CDCcertified facilities in the Galveston National Laboratory at UTMB, Galveston, TX, using established procedures and precautions. S. aureus (ACTT25923) was maintained in laboratories of the Department of Laboratory Diagnostics, Changhai Hospital, Shanghai, China.

\section{Mouse in vivo, anatomically based, quantitative rickettsial adhesion measuring system}

The C57BL/6 mouse- $R$. australis model is an established animal model of human SFG rickettsiosis because the pathology involves disseminated endothelial infection and pathological lesions, including vasculitis in multiple organs, similar to what is observed in human SFG rickettsiosis $[25,38]$. To quantitatively assess rickettsial adhesion in vivo, after an ordinarily lethal dose of $R$. australis $(1 \times$ $10^{7} \mathrm{PFU} / 0.2 \mu \mathrm{l}$; the $\mathrm{LD}_{50}$ is $1 \times 10^{6} \mathrm{PFU}$ ) was injected through the tail vein [38], rickettsial virulence (by plaque assay) was measured in a $1 \mu \mathrm{l}$ blood sample collected from the orbital venous sinus (OVS) at different times until $1 \mathrm{~h}$ post infection (p.i.). Taking advantage of this anatomically based inoculation model, the principle concept is that the more rickettsiae adhered to the luminal surfaces of the blood vessels, the fewer rickettsiae could be detected in blood samples. Multiple visceral organs were then fixed without perfusion rinse (to keep blood in the vessel lumens) for IFbased histological studies to identify unattached rickettsiae, which were trapped in clots in the vessel lumens. Since borders between blood and ECs were invisible in some capillaries, IF-based assessment will exclude capillaries in all organs. For the IF assay, tissue samples of brain and liver were fixed in a $4 \%$ neutral buffered solution of formaldehyde, embedded in paraffin, sectioned at 5- $\mu \mathrm{m}$ thickness, and processed by IF microscopy for detection of SFG rickettsiae as described previously [53]. Briefly, deparaffinized and rehydrated sections were incubated with rabbit or guinea pig polyclonal antibody against SFG rickettsiae $(1: 1000)$ overnight at $4{ }^{\circ} \mathrm{C}$. Rickettsial antigens were detected with AlexaFluor 594-conjugated goat anti-rabbit or DyLight 594-conjugated goat anti-guinea pig antibodies.
Nuclei were counterstained with DAPI. Fluorescent images were analyzed using an Olympus BX51 epifluorescence or Olympus IX81 confocal microscope.

\section{AFM system}

The biomechanical properties of interactions between reOmpB and reANXA2 at protein-protein and protein-cell levels, respectively, were studied using an AFM system (Flex-AFM, Nanosurf AG, Liestal, Switzerland). Colloidal cantilevers with a $5-\mu \mathrm{m}$ polystyrene bead were used (SHOCON-G-PS, Applied NanoStructures, Mountain View, CA). Published protocols were employed to coat polystyrene beads and polystyrene slides (Electron Microscopy Sciences, Hatfield, PA) with streptavidin [54] and biotinylize recombinant proteins [55]. The cantilevers were functionalized by incubation with streptavidin-reOmpB at $50 \mu \mathrm{g} / \mathrm{ml}$ overnight at $4{ }^{\circ} \mathrm{C}$. The polystyrene slides were functionalized by incubation with streptavidin-reANXA2 or Plg at $50 \mu \mathrm{g} / \mathrm{ml}$ overnight at $4{ }^{\circ} \mathrm{C}$. Unbound proteins were rinsed away using PBS. The exposed surface of the bead was blocked by BSA (Sigma, St. Louis, MO) at $500 \mu \mathrm{g} / \mathrm{ml}$ in PBS [53]. AFM imaging and measurements were generally taken within $1 \mathrm{~h}$ after blocking. The spring constant of the cantilever was calibrated using the Sader method in air [56]. The cantilever spring constant varied between $0.10-0.15 \mathrm{~N} / \mathrm{m}$. Force spectroscopy was done in static force mode operating on $25 \mu \mathrm{m}^{2}$ areas on a living cell surface or on a recombinant protein-functionalized slide to measure the specific unbinding force during rupture of the protein-cell or protein-protein interaction. The maximum compression force was set to $150 \mathrm{pN}$. The contact time was kept constantly at $500 \mathrm{~ms}$ before the cantilever was retracted at a constant pulling speed of $1 \mu \mathrm{m} / \mathrm{s}$ to measure the force-extension curve. In order to evaluate the effect of the nonspecific unbinding force, confluent cell monolayers were pretreated with anti-ANXA2 antibody at $3 \mu \mathrm{g} / \mathrm{ml}$ for 30 min to block ANXA2 on the cell surface before the AFM measurements. Normal mouse IgG was employed as negative control. We scanned five cells per group, each with a different cantilever. FD curves were analyzed using the open source software Atomic J [57].

All other methods are detailed in the Supplementary Materials section.

\section{Statistical analysis}

Values are reported as mean \pm SEM. The data were analyzed using Student's $t$ test or one-way ANOVA analysis (Sigmaplot, Sigma Stat, Jandel Scientific Software, San Rafael, CA). $P$ values are as follows: $* * P<0.01$ and $* P<$ 0.05 . Statistical significance was considered as $P<0.05$. 


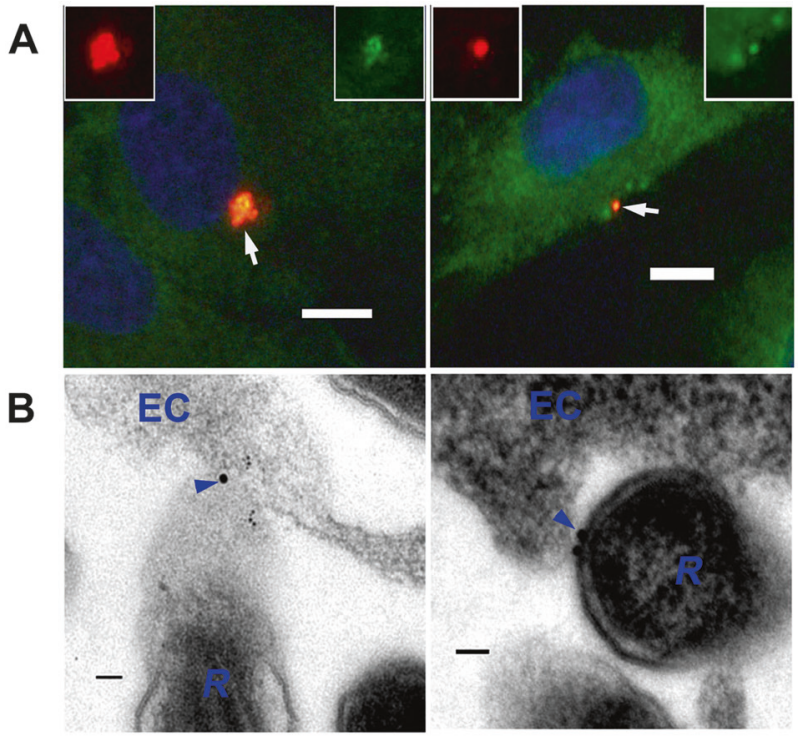

Fig. 1 ANXA2 is involved in rickettsial attachment to nonphagocytic host ECs. a Representative dual-target IF staining of rickettsiae (red) and ANXA2 (green) in HUVECs 15 min post infection (p.i.) with $R$. australis at 10 MOI. HUVECs were fixed with $4 \%$ paraformaldehyde as an impermeable fixation. Nuclei of HUVECs were counterstained with DAPI (blue). The areas indicated by the arrows are enlarged and distinguish rickettsial (red) and ANXA2 (green) staining (boxed inserts). Scale bars, $10 \mu \mathrm{m}$. b Immunoelectron microscopic (IEM) detection of ANXA2 (arrowheads) in HUVECs (EC) infected with $R$. australis $(\mathrm{R}) 15$ min p.i. with $R$. australis at 10 MOI. Scale bars, $100 \mathrm{~nm}$

\section{Results}

\section{Rickettsiae associated with ANXA2 on EC surfaces upon initial infection}

ANXA2 on the surfaces of HUVECs at the beginning of infection with $R$. australis at $10 \mathrm{MOI}$ was visualized in vitro using IF confocal microscopy on nonpermeabilized, fixed cells [38, 58, 59]. IF staining revealed that ANXA2 (Figs. 1a and S1) colocalized with rickettsiae on plasma membrane external surfaces. Immunoelectron microscopy (IEM) studies using anti-ANXA2 antibodies demonstrated that ANXA2 was detected between the surfaces of rickettsiae and the external surfaces of ECs shortly after infection (Fig. 1b).

These data demonstrate that ANXA2 on apical surfaces of ECs appears to participate in establishing rickettisal attachment on EC surfaces.

\section{Deletion of ANXA2 impeded rickettsial attachment to the ECs in vitro}

Growing evidence indicates that mammalian cell surface ANXA2 participates in heterotypic cell-to-cell adhesion $[60,61]$. It is further emerging that ANXA2 is involved in
A

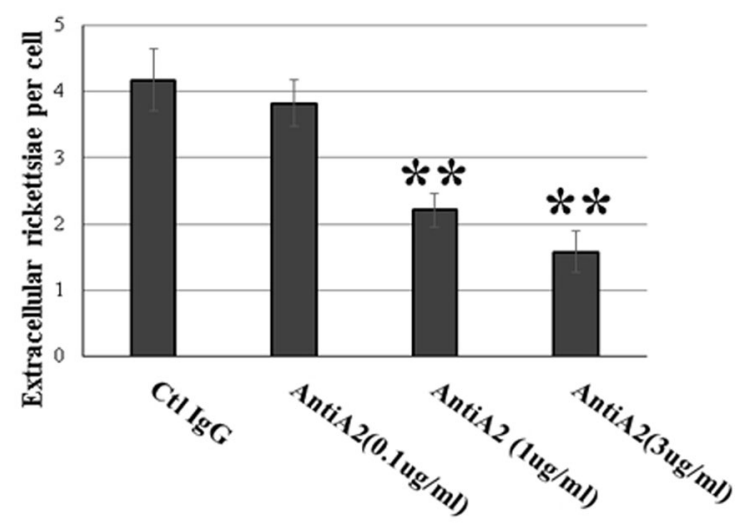

B

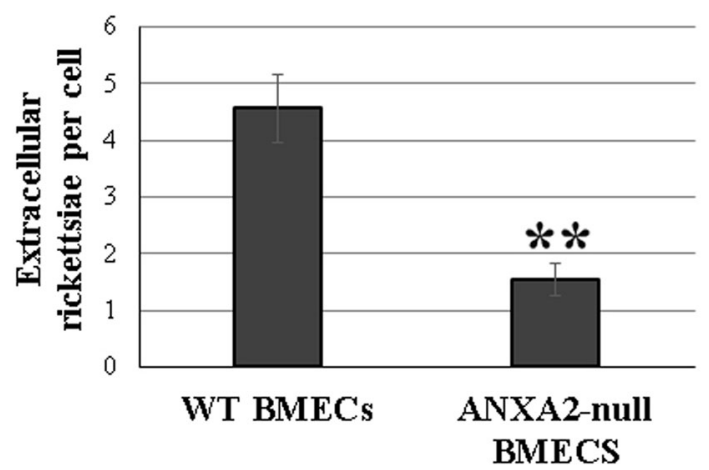

Fig. 2 Inactivation of ANXA2 impeded rickettsial attachment to the endothelial cells in vitro. Extracellular adhesive bacteria in antiANXA2 (AntiA2)-treated HUVECs at $0.1(n=5), 1(n=10)$, and 3 $(n=5) \mu \mathrm{g} / \mathrm{ml}(\mathbf{a})$ or BMECs from WT $(n=6)$ and Anxa2-null $(n=6)$ mice (b) were enumerated by IF microscopy at $15 \mathrm{~min}$ p.i. with $R$. australis at 10 MOI. Normal mouse IgG was used as control $\mathrm{IgG}(\mathrm{Ctl}$ $\mathrm{IgG}, 3 \mu \mathrm{g} / \mathrm{ml})(n=5)$. All ECs were fixed with $4 \%$ paraformaldehyde as impermeable fixation. Data are represented as mean \pm SEM. **compared to the control group, $P<0.01$

bacterial invasion $[47,49,51]$. These finding prompted us to determine the potential role of ANXA2 during rickettsial adhesion.

First, we examined whether ANXA2 antibody affects rickettsial adhesion to the endothelial surface. Following pretreatment with an ANXA2 antibody (clone 666316), HUVEC surface-associated rickettsiae were markedly decreased at $15 \mathrm{~min}$ p.i. in a dose-dependent manner, compared to irrelevant normal $\mathrm{IgG}$ controls as observed by fluorescent microscopy (Fig. 2a).

To confirm whether lack of ANXA2 directly impedes bacterial infection, we utilized mouse brain microvascular endothelial cells (BMECs) from WT and Anxa2-null mice. Endothelial surface-associated rickettsiae examined by impermeable IF microscopy were decreased in Anxa2-null BMECS, compared to WT BMECs, at 15 min p.i. with 10 MOI rickettsiae (Fig. 2b).

This in vitro evidence suggests that host ANXA2 plays an imperative role during rickettsial adhesion to host EC surfaces. 
Fig. 3 Global depletion of ANXA2 blocked rickettsial adherence to the blood vessel luminal surface in vivo. a The anatomically based in vivo quantitative rickettsial $(R$.) adhesion analysis system. b Plaque assay for $R$. australis using blood samples collected from the orbital venous sinus of WT $(n=10)$ and ANXA2-null $(n=10)$ mice at different time $\mathrm{p}$. i. with 10 LD50 doses of $R$. australis intravenously. Data are represented as mean \pm SEM.

**Compared to the control group, $P<0.01$. c

Representative IF-based identification of rickettsial (red) location in WT and Anxa2-null mice 60 min p.i. with 10 LD50 doses of $R$. australis

intravenously. Rickettsiae are adhered to intima layer of blood vessels (arrows) or wrapped in blood clots (arrowheads) in the lumens of blood vessels. Nuclei are blue. Scale bars; $20 \mu \mathrm{m}$
A

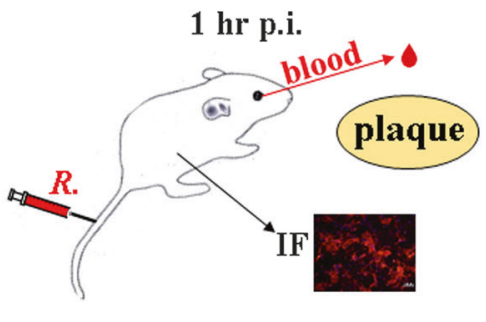

B

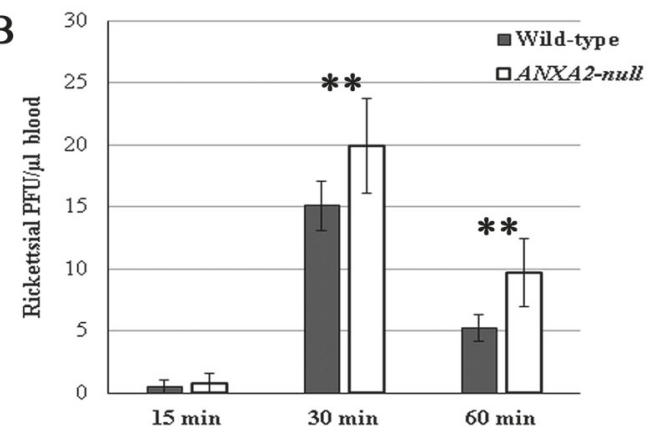

C

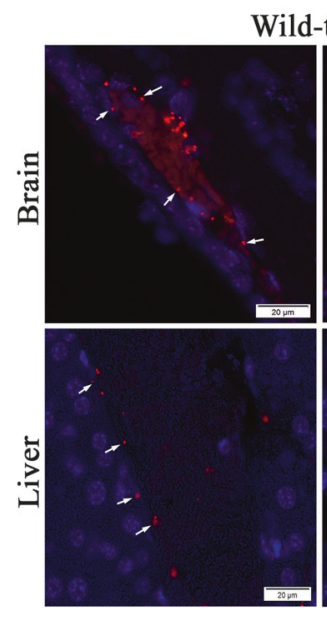

Wild-type
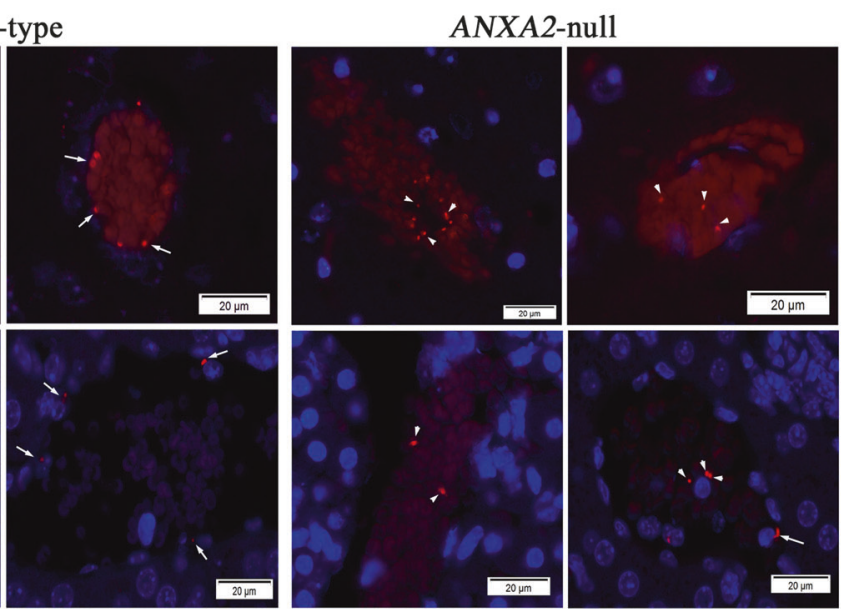

\section{Global depletion of ANXA2 blocked rickettsial adherence to the blood vessel luminal surface in vivo}

After 10 LD50 doses of $R$. australis $\left(1 \times 10^{7}\right.$ PFU) were injected through the tail vein, viable rickettsiae in circulating blood collected from the OVS at different times p.i. was measured by plaque assay (Fig. 3a). Viable bacteria that were not adhered to the endothelium were not detected in blood samples as shown by plaque assay at $15 \mathrm{~min}$ p.i., but they became detectable as early as $30 \mathrm{~min}$ p.i. Plaque assays showed that the number of viable rickettsiae in circulating blood was higher in Anxa2-null mice $(n=10)$ than WT mice $(n=10)$ at $1 \mathrm{~h}$ p.i. (Fig. $3 \mathrm{~b})$.

To keep blood in the vessel lumens in tissue samples, multiple visceral organs were fixed without perfusion for IF-based histological studies to identify unattached rickettsiae that otherwise become trapped in clots in the vessel lumens. IF staining showed that rickettsiae were located in two major blood vessel compartments, the luminal surface or tunica intima of the vascular wall and postmortem artifacts of fixed blood clots that show evidence of trapped circulating bacteria (Fig. 3c). In WT mice $(n=10)$, rickettsiae were detected on the luminal surface or tunica intima of the vascular wall (Figs. 3c and S2-5). However, in Anxa2-null mice $(n=10)$, significantly fewer rickettsiae were detected in the same areas, while unattached rickettsiae were visible in the blood clots in the lumen of these blood vessels (Figs. 3c and S2-5).

The observation that the vascular endothelium in Anxa2null mice does not contain SFG rickettsiae shortly after infection (adherent and invading), coupled with in vitro evidence that deletion of host Anxa2 impedes rickettsial attachment to the endothelial surface, suggests that host ANXA2 plays an important role during rickettsial adhesion to the EC surface.

Interestingly, between the WT and Anxa2-null groups, there was no difference in the quantity of rickettsial antigenpositive white blood cells that were isolated from peripheral blood samples $1 \mathrm{~h}$ following a lethal dose of rickettsiae injected via the tail vein, as detected using flow cytometry (Supplemental Fig. S6).

\section{reANXA2 showed strong interaction with reOmpB}

It remains unclear whether ANXA2 functions as a receptor for direct rickettsial binding or only as a signaling pathway modulator that regulates the mechanical interaction between rickettsial adhesin(s) and other 
A
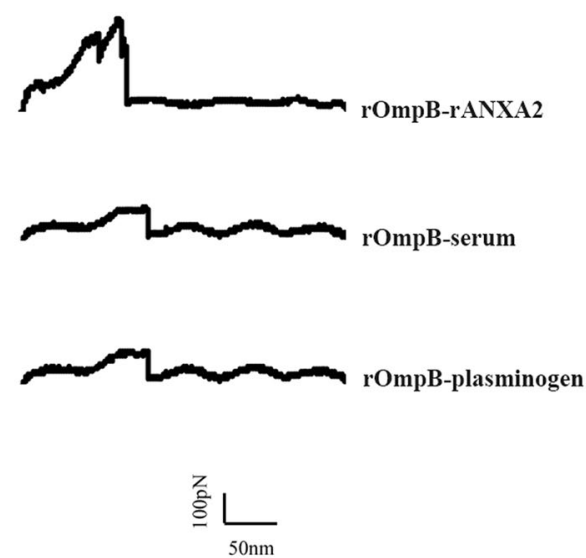

B

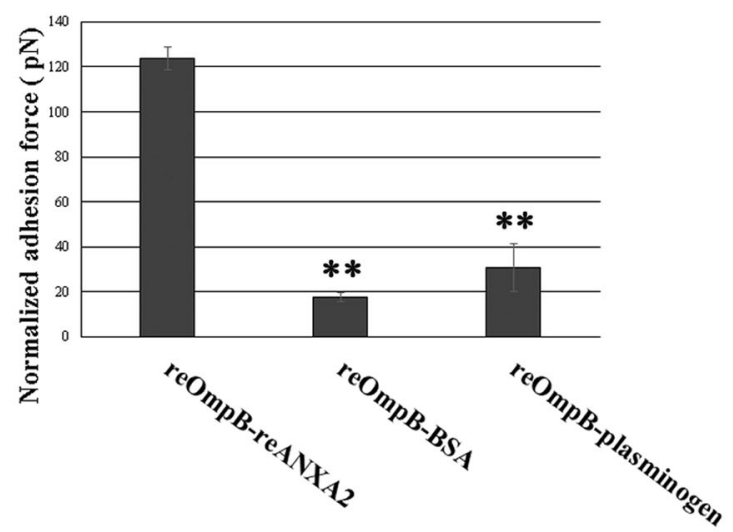

Fig. 4 a Representative force-distance curves were recorded during protein-protein or protein-cell surface separation using an AFM system. The last unbinding event detected is a measure of the unbinding force of the interaction at single molecule level [62]. b AFM studies measured the binding forces between reOmpB and reANXA2, human plasminogen, and BSA, respectively. Data are represented as mean \pm SEM. ${ }^{* *}$ Compared to the group of reANXA2, $P<0.01$. c AFM studies measured the binding forces between reOmpB and HUVECs in normal media, media supplemented with ANXA2 antibodies or normal

unidentified endothelial surface receptor(s). AFM has been increasingly employed in the investigation of the biomechanical basis of bacterial adhesion to host surfaces at the single molecule level, providing deeper insights into the binding mechanism $[18,62,63]$. We functionalized the AFM cantilever probe with reOmpB (amino acids 1363-1655). We conducted the force spectroscopy to directly measure the specific unbinding force during rupture of the interaction between reOmpB and human reANXA2 and Plg. BSA was used as a control. Representative force-distance (FD) curves exhibit rupture events during separation of protein-protein interactions (Fig. 4a). As shown in Fig. 4b, the AFM study using the reOmpB-functionalized probe established that the strongest binding interaction was observed with reANXA2, compared to BSA or Plg.
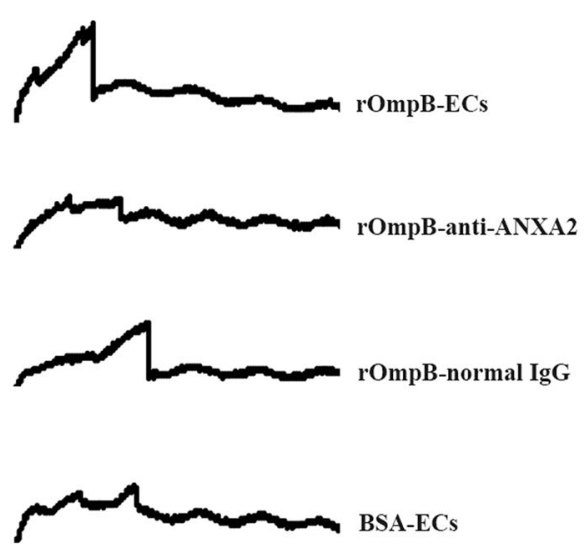

$\mathrm{C}$

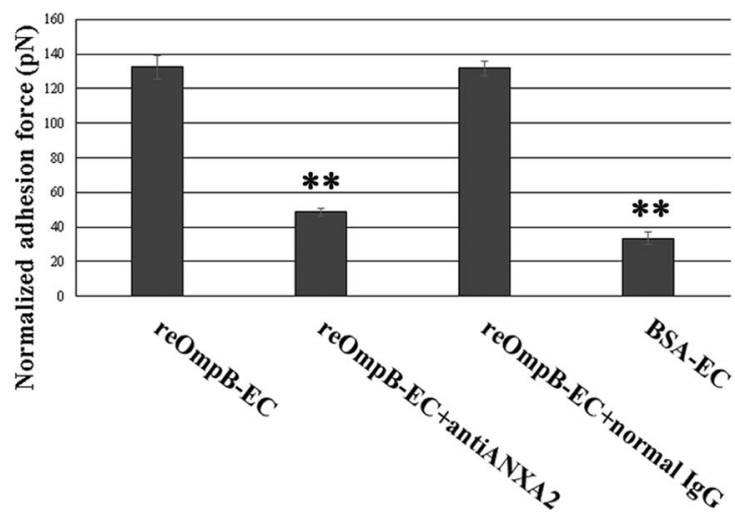

IgG. BSA was employed as a control. Each treatment group was measured in static mode operating on $1 \times 1 \mu \mathrm{m}$ areas $(1024$ data points) of one single living HUVEC for a given period $(1 \mathrm{~h})$. Data acquired during this time for a specific area was regarded as one group data. At least five different detection areas on five different living HUVECs were measured in one treatment group in one experiment. Each experiment was studied in triplicate. Data are represented as mean \pm SEM. $* *$ Compared to the group of normal media, $P<0.01$

\section{reOmpB showed an ANXA2-dependent binding strength on the surface of a live EC}

We employed AFM to directly measure the rickettsial adhesin molecule's binding force to a living host cell surface. Using relevant antibodies as specific functional blockers, the mechanical strength of the interactions between reOmpB and potential receptor(s) expressed at the apical surface of a single living EC can be detected. We applied a reOmpB-coated cantilever probe directly on a single, live HUVEC and found that reOmpB establishes stronger binding to the endothelial surface (Fig. 4c), compared to the BSA-coated probe. Pretreating the HUVEC with an ANXA2 antibody (clone 666316) in media significantly reduced the unbinding force between the reOmpB functionalized AFM probe and the EC surface (Fig. 4c), 
suggesting that reOmpB binds to the surface of a live EC in an ANXA2-dependent manner.

The data from these experiments indicate that ANXA2 on the host EC surface acts as an adherence receptor for $\mathrm{SFG}$ rickettsia, and that $\mathrm{OmpB}$ is the associated bacterial ligand.

\section{Pretreatment of ECs with anti-ANXA2 antibody reduced EC surface-associated $S$. aureus}

To determine the roles that ANXA2 plays in establishing infection of ECs by other bacteria, we examined whether ANXA2 antibody affected binding of $S$. aureus, a facultative intracellular bacterium, to human ECs. We found that, following pretreatment of HUVECs with anti-ANXA2 antibody (clone 666316), the number of endothelial surface-associated $S$. aureus were decreased at $15 \mathrm{~min}$ p.i., compared to irrelevant normal IgG controls, in a dosedependent manner (Fig. 5).

\section{Discussion}

Rickettsial infection can be controlled by appropriate broadspectrum antibiotic therapy after early diagnosis [20, 22, 23, 64]. Nevertheless, untreated or misdiagnosed rickettsioses are frequently associated with severe morbidity and mortality [20, 23, 65-67]. An initial flu-like, nonspecific, clinical febrile presentation partially explains the underdiagnosis of these diseases [68]. A case-fatality rate as high as $32 \%$ has been reported in hospitalized patients with Mediterranean spotted fever [67]. Disseminated endothelial

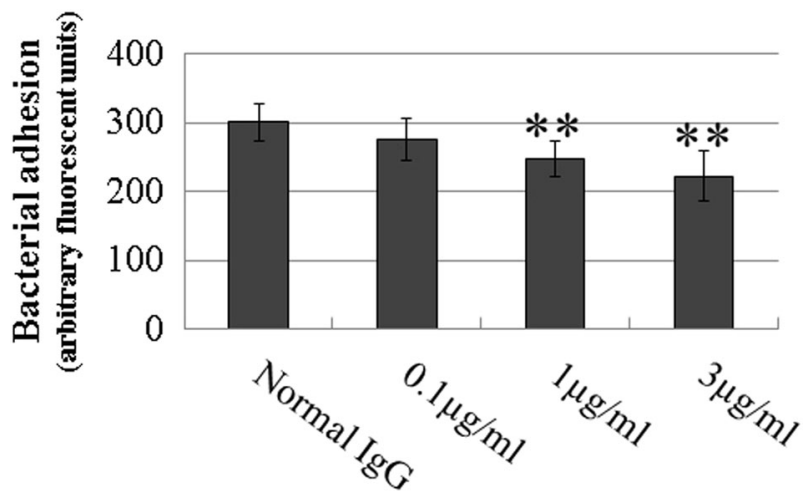

Fig. 5 Pretreatment of HUVECs with ANXA2 antibody reduced endothelial surface-associated $S$. aureus. Extracellular adhesive FITCd-Lys-labeled bacteria in anti-ANXA2 antibody-treated HUVECs at $0.1,1$, and $3 \mu \mathrm{g} / \mathrm{ml}$ were detected by a SpectraMax i3x fluorescence plate reader at $15 \mathrm{~min}$ p.i. with $S$. aureus at $100 \mathrm{MOI}$. Normal mouse $\mathrm{IgG}$ was used as control $\mathrm{IgG}$ (normal $\mathrm{IgG}, 3 \mu \mathrm{g} / \mathrm{ml}$ ). All ECs were fixed with $4 \%$ paraformaldehyde as impermeable fixation. All experiments were repeated three times. There are 35 samples in each group. Data are represented as mean \pm SEM. **Compared to the control group, $P<0.01$ infection and endothelial barrier disruption with increased microvascular permeability are the central pathophysiologic features of rickettsial infection. Rickettsial pathogens exert their pathologic effects after adhering to and then invading the endothelial lining of blood vessels. The bacteria must establish adhesive interactions to firmly anchor on the endothelial luminal surface to successfully resist detachment by hemodynamic shear stress from flowing blood prior to invasion of the EC $[11,27]$. This adherence requires recognition and interaction with specific cellular receptors, and is thought to be dependent mainly on rickettsial outer membrane proteins OmpA (Sca0), Sca1, Sca2, and OmpB (Sca5), which are encoded by surface cell antigen (sca) family genes [29, 58] and expressed on the surface of SFG rickettsiae [69]. These proteins are considered to play important roles as adhesins by mediating interactions with mammalian cells during rickettsial adhesion and subsequent invasion [29, 30, 37]. Our current study unveiled an association between rickettsiae and host ANXA2 on EC surfaces at the beginning of infection. By developing a novel, anatomically based, quantitative bacterial adhesion measuring system, which couples plaque assays and IF, we revealed that global depletion of $A N X A 2$ blocked rickettsial adherence to the blood vessel luminal surface in vivo. Using AFM, we demonstrated that the binding strength of reOmpB with surface ANXA2 in living ECs reflects a direct interaction. Interestingly, an anti-ANXA2 antibody also attenuated $S$. aureus adhesion to the EC surface. Taken together, these findings support that host ANXA2 can serves as an adherence receptor for both rickettsiae and $S$. aureus.

The establishment of bacterium-endothelium adhesion is firm enough to withstand shear forces from blood flow, and requires specific host cell surface receptors that recognize and interact with bacterial adhesin(s). Different elements on the host plasma membrane external surfaces, including cadherins [70], CD31 [71], fibrinogen [72-74], ICAM-1 [75], integrins [7, 29, 76], Plg [77], platelet activating factor receptor [78], selectins [79], and von Willebrand factor $[11,27]$ are recognized as participating in adhesion by various nonobligately intracellular bacterial pathogens. Our previous study combining genetic and pharmacological manipulations demonstrated that inhibition of intracellular cAMP receptor EPAC1 suppressed rickettsial adherence to ECs [38], although the underlying mechanism remains unclear. Host $\alpha 2 \beta 1$ integrin heterodimer has been identified to be involved in the initial adhesion of $R$. conorii to the host cell [29]. Using IF assays on nonpermeabilized cells, host $\alpha 2 \beta 1$ integrin heterodimer has been identified as an adherence receptor for OmpA heterologously expressed in E. coli. In contrast, OmpB-mediated adhesion is independent of $\alpha 2 \beta 1$ integrin [29]. Furthermore, either functional blocking using antibodies against $\alpha 2 \beta 1$ integrin or small 
interfering RNA (siRNA) transfection that silences $\alpha 2 \beta 1$ integrin synthesis in host cells perturbs $R$. conorii invasion, but does not affect adhesion [29]. Therefore, there must be an unknown host mechanism(s) that allows rickettsiae to interact with host cell surfaces by establishing adhesion prior to invasion.

A critical feature of any host cell receptor for initial bacterial adhesion is its integral location and availability on the vascular luminal surface of ECs, which are the primary targets of rickettsiae present in the circulation following inoculation and subsequent multiplication. Identified host receptors for adhesion of other bacteria are distributed predominantly on the basolateral surfaces of ECs for homologous cell-cell adhesion or cell-extracellular matrix interactions, or reside inside the cell and are translocated to the cell surface during an inflammatory response [7, 11, 27, 29, 70-76, 79]. For rickettsiae, proteins in host subcellular compartments may potentially participate in host-pathogen interactions during subsequent invasion, rather than during initial attachment.

ANXA2 is an important receptor that allows Plg and tPA to assemble host fibrinolytic machinery on the endothelial luminal surface, and plays a critical effector role in vascular patency $[45,80,81]$. Lines of evidence from the present study unveiled a novel role for endothelial luminal surface ANXA2, which serves as a host cell-binding unit for rickettsial adhesion. Global abolishment of ANXA2 in vivo decreases the number of rickettsiae attached to the vascular luminal surfaces, but increases the number of bacteria in circulating blood at the initial stages of infection following intravenous inoculation. Pretreatment of ECs with antiANXA2 antibody also reduced endothelial surfaceassociated S. aureus. An AFM study provided direct evidence that endothelial ANXA2 is an adherence receptor for rickettsial binding on the endothelial surface.

In bacteriology, it has been reported that in vitro experiments utilizing static conditions and dynamic experimental flow conditions can lead to different outcomes for bacterial adherence to ECs [74, 82]. To the best of our knowledge, in rickettsiology there have been no reports on approaches that take into account hemodynamic forces [83]; there have been no mechanistic studies to identify adherence receptors by examining the rickettsia-host receptor binding force that is able to withstand hemodynamic shear stresses in vitro or in vivo. These facts highlight the importance of studying rickettsial adhesion mechanisms using real-time measurements of nanoscale microforces of interactions between rickettsial adhesins and endothelial surfaces. AFM techniques are particularly well suited to achieve this goal [62]. The key advantage of AFM over other imaging techniques in this study is that it provides a measurement of real-time, nanoscale-relevant information of the interactive forces between a single ligand and its receptor [63]. Over the last two decades, this technique has been developed to measure inter- and intra-molecular forces, revealing detailed insights into the functional mechanics of biomolecules. By using AFM on living cells infected with Listeria monocytogenes, the unbinding force between a bacterial ligand and its host receptor were measured [63]. In the present study, we employed AFM in protein-protein and protein-living cell models, and obtained nanobiomechanical information to help elucidate the rickettsiae-host cell surface interaction that allows the rickettsiae to overcome shear stress within a blood vessel and establish a productive infection.

Previous studies have identified specific rickettsial proteins as adhesins, particularly the OmpA and OmpB [29, 35, 84]. Antibodies to OmpA and OmpB, but not to lipopolysaccharide, can protect severe combined immunodeficiency mice against fatal SFG rickettsial infection [85]. OmpB is the most abundant protein that is expressed in the outer membrane, and occurs in all rickettsial species [86]. Rickettsial OmpB can be cleaved into a passenger domain (amino acids 451-1308) and the $\beta$-peptide (amino acids 1335-1704) [86, 87]. The passenger domain has been reported to be functional in bacterial invasion by interaction with $\mathrm{Ku} 70$ [37], while the $\beta$-peptide has been shown to interact with mammalian surface proteins [35]. Our AFM study on protein-protein interactions showed that reOmpB (amino acids 1363-1655) binds to reANXA2 significantly in vitro. By using AFM in a protein-living cell interaction model, we probed direct reOmpB binding to living endothelial apical surfaces in an ANXA2-dependent manner. These data suggest that the rickettsial OmpB-ANAX2 interaction exists as a mechanic force during rickettsial binding to the host EC surface. OmpB is the rickettsial effector in establishing binding to ANXA2. This has prompted us to plan experiments using the same approaches to identify potential adhesin(s) of $S$. aureus, which lacks outer membrane proteins, in the interaction with ANAX2.

Importantly, current state-of-the-art in vitro models have been successful for elucidating mechanisms regarding bacterial adhesion to host cells, and have significantly improved outcomes following genetic manipulation of selected targets [36, 38]. However, there are deficiencies using in vitro models to evaluate bacterial adhesion to endothelium [74, 82]. In order for rickettsiae to invade cells, the bacteria must first attach to the EC surface that is under hemodynamic shear stress, which occurs at the blood vessel luminal surface in the direction of blood flow [88]. Although a closed-pump system can be used as an in vitro model mimicking dynamic circulation, such an approach poorly reflects the physiological hemodynamic forces and the anatomical complexity of the endothelium system in vivo and imposes significant limitations for downstream experiments. To date, surgical- and intravital fluorescent 
microscopy-based rodent models have been used in kinetic studies of Staphylococcus adhesion to endothelium in vivo $[11,12]$. In addition, mouse models are emerging as a useful model system in postmortem histopathology- or homogenized tissue-based in vivo studies on bacterial infection involving adhesion and invasion [13, 89-91]. Nevertheless, there have been no reports that describe mouse in vivo approaches that take into account physiological hemodynamic forces and genetic manipulation of biochemical targets using mutant strains [19]. Using established Anxa2-null mice, we have developed a novel anatomically based, in vivo quantitative bacterial adhesion measuring system, which couples plaque assays and IF, and provides a platform to meet the sampling requirements for more downstream experimental approaches, e.g., quantitative real-time PCR and electron microscopy. This system allows us to quantitatively evaluate bacterial adherence to blood vessel luminal surfaces under physiological hemodynamic shear forces using C57BL/6J WT mice and global Anxa2-null mice. To our knowledge, this is the first noninvasive mouse model for quantitative in vivo studies of pathogen adhesion to endothelium in a physiologically simulated hemodynamic environment.

In conclusion, our studies combined an AFM protein-protein and protein-living cell interacting force measuring system with an in vivo model that allowed us to study bacterial adhesion to the vascular EC. This work revealed a novel role for endothelial surface ANXA2 as a receptor for SFG rickettsial binding. Rickettsial OmpB is the bacterial ligand for this interaction. Endothelial ANXA2 is also involved in establishing $S$. aureus binding to the EC surface. Therefore, endothelial apical surface ANXA2 plays an important role in initiating pathogen-host interactions, ultimately leading to bacterial anchoring on the vascular luminal surface.

Acknowledgements We gratefully acknowledge Dr Donald Bouyer for support during BSL3 experiments. We would like to thank Dr. Katherine Hajjar for the gift of Anxa2-null mice. We thank Drs Juan Olano and Lucas Blanton for supporting reagents to facilitate immunostaining. We gratefully acknowledge Drs Kimberly Schuenke and David Walker for their critical reviews and editing of the manuscript. We thank Nicole Mendel and Thomas Shelite for technical support during initiation of the in vivo model. We thank Dr Jing Wang, Patricia Valdes, Yue Qu, Ben Zhang, Xiang Li, Yuejing Liang, Junying Zheng, Kayla Bayless, and Zhixia Ding for technical assistances. We thank Eric Gong for his preparation of the figures. This work was supported by NIH grant R01AI121012 (BG) and R21AI137785 (BG), and National Natural Science Foundation of China grant 81370265 (F L). The funders had no role in the study design, data collection, and analysis, decision to publish, or preparation of the manuscript.

Funding This work was supported by NIH (BG) and Natural Science Foundation of China (FL).

\section{Compliance with ethical standards}

Conflict of interest The authors declare that they have no conflict of interest.

Publisher's note: Springer Nature remains neutral with regard to jurisdictional claims in published maps and institutional affiliations.

\section{References}

1. Johnson RW, McGillivary G, Denoël P, Poolman J, Bakaletz LO. Abrogation of nontypeable Haemophilus influenzae protein D function reduces phosphorylcholine decoration, adherence to airway epithelial cells, and fitness in a chinchilla model of otitis media. Vaccine. 2011;29:1211-21.

2. Bruckert WM, Price CT, Abu Kwaik Y. Rapid nutritional remodeling of the host cell upon attachment of Legionella pneumophila. Infect Immun. 2014;82:72-82.

3. Caine JA, Lin YP, Kessler JR, Sato H, Leong JM, Coburn J. Borrelia burgdorferi outer surface protein $\mathrm{C}(\mathrm{OspC})$ binds complement component $\mathrm{C} 4 \mathrm{~b}$ and confers bloodstream survival. Cell Microbiol. 2017;19:e12786.

4. Darville T, Hiltke TJ. Pathogenesis of genital tract disease due to Chlamydia trachomatis. J Infect Dis. 2010;201:S114-25.

5. Williams MA, Schmidt RL, Lenz LL. Early events regulating immunity and pathogenesis during Listeria monocytogenes infection. Trends Immunol. 2012;33:488-95.

6. Maldonado-Arocho FJ, Green C, Fisher ML, Paczosa MK, Mecsas J. Adhesins and host serum factors drive Yop translocation by yersinia into professional phagocytes during animal infection. PLoS Pathog. 2013;9:e1003415.

7. Orihuela CJ, Mahdavi J, Thornton J, Mann B, Wooldridge KG, Abouseada $\mathrm{N}$, et al. Laminin receptor initiates bacterial contact with the blood brain barrier in experimental meningitis models. $\mathrm{J}$ Clin Investig. 2009;119:1638-46.

8. Rasmussen JW, Tam JW, Okan NA, Mena P, Furie MB, Thanassi DG, et al. Phenotypic, morphological, and functional heterogeneity of splenic immature myeloid cells in the host response to tularemia. Infect Immun. 2012;80:2371-81.

9. Lamason RL, Welch MD. Actin-based motility and cell-to-cell spread of bacterial pathogens. Curr Opin Microbiol. 2017; $35: 48-57$.

10. Coleman JL, Sellati TJ, Testa JE, Kew RR, Furie MB, Benach JL. Borrelia burgdorferi binds plasminogen, resulting in enhanced penetration of endothelial monolayers. Infect Immun. 1995; 63:2478-84.

11. Claes J, Vanassche T, Peetermans M, Liesenborghs L, Vandenbriele C, Vanhoorelbeke K, et al. Adhesion of Staphylococcus aureus to the vessel wall under flow is mediated by von Willebrand factor-binding protein. Blood. 2014;124:1669-76.

12. Kerdudou S, Laschke MW, Sinha B, Preissner KT, Menger MD, Herrmann M. Fibronectin binding proteins contribute to the adherence of Staphylococcus aureus to intact endothelium in vivo. Thromb Haemost. 2006;96:183-9.

13. McMullen PD, Freitag NE. Assessing bacterial invasion of cardiac cells in culture and heart colonization in infected mice using Listeria monocytogenes. J Vis Exp. 2015:e52497.

14. Lee BC, Mayer CL, Leibowitz CS, Stearns-Kurosawa DJ, Kurosawa S. Quiescent complement in nonhuman primates during $E$. coli Shiga toxin-induced hemolytic uremic syndrome and thrombotic microangiopathy. Blood. 2013;122:803-6.

15. Shenoy AT, Beno SM, Brissac T, Bell JW, Novak L, Orihuela CJ. Severity and properties of cardiac damage caused by 
Streptococcus pneumoniae are strain dependent. PLoS ONE. 2018;13:e204032.

16. Mellata M, Mitchell NM, Schödel F, Curtiss R, Pier GB. Novel vaccine antigen combinations elicit protective immune responses against Escherichia coli sepsis. Vaccine. 2016;34:656-62.

17. Viscoli C. Bloodstream infections: the peak of the iceberg. Virulence. 2016;7:248-51.

18. Beaussart A, El-Kirat-Chatel S, Sullan RM, Alsteens D, Herman $\mathrm{P}$, Derclaye S, et al. Quantifying the forces guiding microbial cell adhesion using single-cell force spectroscopy. Nat Protoc. 2014;9:1049-55.

19. Edwards AM, Bowden MG, Brown EL, Laabei M, Massey RC. Staphylococcus aureus extracellular adherence protein triggers TNF $\alpha$ release, promoting attachment to endothelial cells via protein A. PLoS ONE. 2012;7:e43046.

20. Dumler JS, Walker DH. Rocky Mountain spotted fever-changing ecology and persisting virulence. N Engl J Med. 2005;353:551-3.

21. Walker DH, Ismail N. Emerging and re-emerging rickettsioses: endothelial cell infection and early disease events. Nat Rev Microbiol. 2008;6:375-86.

22. Chapman AS, Murphy SM, Demma LJ, Holman RC, Curns AT, McQuiston JH, et al. Rocky mountain spotted fever in the United States, 1997-2002. Ann N Y Acad Sci. 2006;1078:154-5.

23. Walker DH, Paddock CD, Dumler JS. Emerging and re-emerging tick-transmitted rickettsial and ehrlichial infections. Med Clin N Am. 2008;92:1345-61, x.

24. Chan YG, Riley SP, Martinez JJ. Adherence to and invasion of host cells by spotted fever group rickettsia species. Front Microbiol. 2010;1:139.

25. Feng HM, Wen J, Walker DH. Rickettsia australis infection: a murine model of a highly invasive vasculopathic rickettsiosis. Am J Pathol. 1993;142:1471-82.

26. Stewart A, Armstrong M, Graves S, Hajkowicz K, Queensland Tick. Typhus: a rickettsial spotted fever group infection in Australia. Am J Trop Med Hyg. 2017;97:24-9.

27. Pappelbaum KI, Gorzelanny C, Grässle S, Suckau J, Laschke MW, Bischoff M, et al. Ultralarge von Willebrand factor fibers mediate luminal Staphylococcus aureus adhesion to an intact endothelial cell layer under shear stress. Circulation. 2013;128:50-9.

28. Pries AR, Secomb TW, Gaehtgens P. The endothelial surface layer. Pflugers Arch. 2000;440:653-66.

29. Hillman RD, Baktash YM, Martinez JJ. OmpA-mediated rickettsial adherence to and invasion of human endothelial cells is dependent upon interaction with $\alpha 2 \beta 1$ integrin. Cell Microbiol. 2013;15:727-41.

30. Li H, Walker DH. rOmpA is a critical protein for the adhesion of Rickettsia rickettsii to host cells. Microb Pathog. 1998;24:289-98.

31. Uchiyama T, Kawano H, Kusuhara Y. The major outer membrane protein rOmpB of spotted fever group rickettsiae functions in the rickettsial adherence to and invasion of Vero cells. Microbes Infect. 2006;8:801-9.

32. Riley SP, Goh KC, Hermanas TM, Cardwell MM, Chan YG, Martinez JJ. The Rickettsia conorii autotransporter protein Scal promotes adherence to nonphagocytic mammalian cells. Infect Immun. 2010;78:1895-904.

33. Cardwell MM, Martinez JJ. The Sca2 autotransporter protein from Rickettsia conorii is sufficient to mediate adherence to and invasion of cultured mammalian cells. Infect Immun. 2009;77: 5272-80.

34. Vellaiswamy M, Kowalczewska M, Merhej V, Nappez C, Vincentelli R, Renesto P, et al. Characterization of rickettsial adhesin Adr2 belonging to a new group of adhesins in $\alpha$-proteobacteria. Microb Pathog. 2011;50:233-42.

35. Renesto P, Samson L, Ogata H, Azza S, Fourquet P, Gorvel JP, et al. Identification of two putative rickettsial adhesins by proteomic analysis. Res Microbiol. 2006;157:605-12.
36. Martinez JJ, Seveau S, Veiga E, Matsuyama S, Cossart P. Ku70, a component of DNA-dependent protein kinase, is a mammalian receptor for Rickettsia conorii. Cell. 2005;123:1013-23.

37. Chan YG, Cardwell MM, Hermanas TM, Uchiyama T, Martinez JJ. Rickettsial outer-membrane protein $\mathrm{B}(\mathrm{rOmpB})$ mediates bacterial invasion through $\mathrm{Ku} 70$ in an actin, c-Cbl, clathrin and caveolin 2-dependent manner. Cell Microbiol. 2009;11:629-44.

38. Gong B, Shelite T, Mei FC, Ha T, Hu Y, Xu G, et al. Exchange protein directly activated by cAMP plays a critical role in bacterial invasion during fatal rickettsioses. Proc Natl Acad Sci USA. 2013;110:19615-20.

39. Madamanchi A, Santoro SA, Zutter MM. $\alpha 2 \beta 1$ Integrin. Adv Exp Med Biol. 2014;819:41-60.

40. de Rooij J, Zwartkruis FJ, Verheijen MH, Cool RH, Nijman SM, Wittinghofer A, et al. Epac is a Rap1 guanine-nucleotideexchange factor directly activated by cyclic AMP. Nature. 1998;396:474-7.

41. Deanfield JE, Halcox JP, Rabelink TJ. Endothelial function and dysfunction: testing and clinical relevance. Circulation. 2007;115: 1285-95.

42. Liu Y, Myrvang HK, Dekker LV. Annexin A2 complexes with S100 proteins: structure, function and pharmacological manipulation. Br J Pharmacol. 2014;1664-76.

43. Bharadwaj A, Bydoun M, Holloway R, Waisman D. Annexin A2 heterotetramer: structure and function. Int J Mol Sci. 2013;14: 6259-305.

44. Hajjar KA, Jacovina AT, Chacko J. An endothelial cell receptor for plasminogen/tissue plasminogen activator. I. Identity with annexin II. J Biol Chem. 1994;269:21191-7.

45. Luo M, Hajjar KA. Annexin A2 system in human biology: cell surface and beyond. Semin Thromb Hemost. 2013;39:338-46.

46. Ashraf S, Cheng J, Zhao X. Clumping factor A of Staphylococcus aureus interacts with AnnexinA2 on mammary epithelial cells. Sci Rep. 2017;7:40608.

47. Tobe T. Cytoskeleton-modulating effectors of enteropathogenic and enterohemorrhagic Escherichia coli: role of EspL2 in adherence and an alternative pathway for modulating cytoskeleton through Annexin A2 function. FEBS J. 2010;277:2403-8.

48. Munera D, Martinez E, Varyukhina S, Mahajan A, AyalaSanmartin J, Frankel G. Recruitment and membrane interactions of host cell proteins during attachment of enteropathogenic and enterohaemorrhagic Escherichia coli. Biochem J. 2012;445: 383-92.

49. Kirschnek S, Adams C, Gulbins E. Annexin II is a novel receptor for Pseudomonas aeruginosa. Biochem Biophys Res Commun. 2005;327:900-6.

50. Jolly C, Winfree S, Hansen B, Steele-Mortimer O. The Annexin A2/p11 complex is required for efficient invasion of Salmonella Typhimurium in epithelial cells. Cell Microbiol. 2014;16:64-77.

51. Somarajan SR, Al-Asadi F, Ramasamy K, Pandranki L, Baseman JB, Kannan TR Annexin A2 mediates Mycoplasma pneumoniae community-acquired respiratory distress syndrome toxin binding to eukaryotic cells. MBio. 2014;5:e1497-14.

52. Ling Q, Jacovina AT, Deora A, Febbraio M, Simantov R, Silverstein RL, et al. Annexin II regulates fibrin homeostasis and neoangiogenesis in vivo. J Clin Investig. 2004;113:38-48.

53. Gong B, Ma L, Liu Y, Gong Q, Shelite T, Bouyer D, et al. Rickettsiae induce microvascular hyperpermeability via phosphorylation of VE-cadherins: evidence from atomic force microscopy and biochemical studies. PLoS NeglTrop Dis. 2012;6: e1699.

54. Ylikotila J, Välimaa L, Takalo H, Pettersson K. Improved surface stability and biotin binding properties of streptavidin coating on polystyrene. Colloids Surf B Biointerfaces. 2009;70:271-7.

55. Kay BK, Thai S, Volgina VV. High-throughput biotinylation of proteins. Methods Mol Biol. 2009;498:185-96. 
56. Xie H, Yin M, Rong W, Sun L. In situ quantification of living cell adhesion forces: single cell force spectroscopy with a nanotweezer. Langmuir. 2014;30:2952-9.

57. Hermanowicz P, Sarna M, Burda K, Gabryś H. AtomicJ: an open source software for analysis of force curves. Rev Sci Instrum. 2014;85:063703.

58. Cardwell MM, Martinez JJ. Identification and characterization of the mammalian association and actin-nucleating domains in the Rickettsia conorii autotransporter protein, Sca2. Cell Microbiol. 2012;14:1485-95.

59. Deora AB, Kreitzer G, Jacovina AT, Hajjar KA. An annexin 2 phosphorylation switch mediates p11-dependent translocation of annexin 2 to the cell surface. J Biol Chem. 2004;279:43411-8.

60. Myrvang HK, Guo X, Li C, Dekker LV. Protein interactions between surface annexin A2 and S100A10 mediate adhesion of breast cancer cells to microvascular endothelial cells. FEBS Lett. 2013;587:3210-5.

61. Garrido-Gómez T, Dominguez F, Quiñonero A, Estella C, Vilella F, Pellicer A, et al. Annexin A2 is critical for embryo adhesiveness to the human endometrium by RhoA activation through Factin regulation. FASEB J. 2012;26:3715-27.

62. Benoit M, Selhuber-Unkel C. Measuring cell adhesion forces: theory and principles. Methods Mol Biol. 2011;736:355-77.

63. Mostowy S, Janel S, Forestier C, Roduit C, Kasas S, PizarroCerdá $\mathrm{J}$, et al. A role for septins in the interaction between the Listeria monocytogenes INVASION PROTEIN InlB and the Met receptor. Biophys J. 2011;100:1949-59.

64. Mansueto P, Vitale G, Cascio A, Seidita A, Pepe I, Carroccio A, et al. New insight into immunity and immunopathology of Rickettsial diseases. Clin Dev immunol. 2012;2012:967852.

65. Openshaw JJ, Swerdlow DL, Krebs JW, Holman RC, Mandel E, Harvey A, et al. Rocky mountain spotted fever in the United States, 2000-2007: interpreting contemporary increases in incidence. Am J Trop Med Hyg. 2010;83:174-82.

66. Botelho-Nevers E, Socolovschi C, Raoult D, Parola P. Treatment of Rickettsia spp. infections: a review. Expert Rev Anti Infect Ther. 2012;10:1425-37.

67. de Sousa R, Nóbrega SD, Bacellar F, Torgal J. Mediterranean spotted fever in Portugal: risk factors for fatal outcome in 105 hospitalized patients. Ann N Y Acad Sci. 2003;990:285-94.

68. Valbuena G, Walker DH. Infection of the endothelium by members of the order Rickettsiales. Thromb Haemost. 2009;102:1071-9.

69. Henderson IR, Nataro JP. Virulence functions of autotransporter proteins. Infect Immun. 2001;69:1231-43.

70. Bonazzi M, Veiga E, Pizarro-Cerdá J, Cossart P. Successive posttranslational modifications of E-cadherin are required for InlAmediated internalization of Listeria monocytogenes. Cell Microbiol. 2008;10:2208-22.

71. Iovino F, Molema G, Bijlsma JJ. Platelet endothelial cell adhesion molecule-1, a putative receptor for the adhesion of Streptococcus pneumoniae to the vascular endothelium of the blood-brain barrier. Infect Immun. 2014;82:3555-66.

72. Massey RC, Kantzanou MN, Fowler T, Day NP, Schofield K, Wann ER, et al. Fibronectin-binding protein A of Staphylococcus aureus has multiple, substituting, binding regions that mediate adherence to fibronectin and invasion of endothelial cells. Cell Microbiol. 2001;3:839-51.

73. Bedran TB, Azelmat J, Spolidorio DP, Grenier D. Fibrinogeninduced streptococcus mutans biofilm formation and adherence to endothelial cells. Biomed Res Int. 2013;2013:431465.

74. Shenkman B, Varon D, Tamarin I, Dardik R, Peisachov M, Savion $\mathrm{N}$, et al. Role of agr (RNAIII) in Staphylococcus aureus adherence to fibrinogen, fibronectin, platelets and endothelial cells under static and flow conditions. J Med Microbiol. 2002;51:747-54.
75. Mao S, Maeno N, Matayoshi S, Yoshiie K, Fujimura T, Oda H. The induction of intercellular adhesion molecule-1 on human umbilical vein endothelial cells by a heat-stable component of Porphyromonas gingivalis. Curr Microbiol. 2004;48:108-12.

76. Behera AK, Hildebrand E, Uematsu S, Akira S, Coburn J, Hu LT. Identification of a TLR-independent pathway for Borrelia burgdorferi-induced expression of matrix metalloproteinases and inflammatory mediators through binding to integrin alpha 3 beta 1 . J Immunol. 2006;177:657-64.

77. Bergmann S, Schoenen H, Hammerschmidt S. The interaction between bacterial enolase and plasminogen promotes adherence of Streptococcus pneumoniae to epithelial and endothelial cells. Int J Med Microbiol. 2013;303:452-62.

78. Swords WE, Buscher BA, Ver Steeg Ii K, Preston A, Nichols WA, Weiser JN, et al. Non-typeable Haemophilus influenzae adhere to and invade human bronchial epithelial cells via an interaction of lipooligosaccharide with the PAF receptor. Mol Microbiol. 2000;37:13-27.

79. Komatsu T, Nagano K, Sugiura S, Hagiwara M, Tanigawa N, Abiko Y, et al. E-selectin mediates Porphyromonas gingivalis adherence to human endothelial cells. Infect Immun. 2012;80: 2570-6.

80. Chapin JC, Hajjar KA. Fibrinolysis and the control of blood coagulation. Blood Rev. 2014;17-24.

81. Reddy TR, Li C, Guo X, Fischer PM, Dekker LV. Design. synthesis and SAR exploration of tri-substituted 1,2,4-triazoles as inhibitors of the annexin A2-S100A10 protein interaction. Bioorg Med Chem. 2014;22:5378-91.

82. Müller NF, Kaiser PO, Linke D, Schwarz H, Riess T, Schäfer A, et al. Trimeric autotransporter adhesin-dependent adherence of Bartonella henselae, Bartonella quintana, and Yersinia enterocolitica to matrix components and endothelial cells under static and dynamic flow conditions. Infect Immun. 2011;79:2544-53.

83. Valbuena G, Walker DH. The endothelium as a target for infections. Annu Rev Pathol. 2006;1:171-98.

84. Noriea NF, Clark TR, Mead D, Hackstadt T. Proteolytic cleavage of the immunodominant outer membrane protein rOmpA in Rickettsia rickettsii. J Bacteriol. 2017;199:e00826-16.

85. Feng HM, Whitworth T, Olano JP, Popov VL, Walker DH. Fcdependent polyclonal antibodies and antibodies to outer membrane proteins $\mathrm{A}$ and $\mathrm{B}$, but not to lipopolysaccharide, protect SCID mice against fatal Rickettsia conorii infection. Infect Immun. 2004;72:2222-8.

86. Chan YG, Riley SP, Chen E, Martinez JJ. Molecular basis of immunity to rickettsial infection conferred through outer membrane protein B. Infect Immun. 2011;79:2303-13.

87. Díaz-Montero CM, Feng HM, Crocquet-Valdes PA, Walker DH. Identification of protective components of two major outer membrane proteins of spotted fever group Rickettsiae. Am J Trop Med Hyg. 2001;65:371-8.

88. Davies PF. Hemodynamic shear stress and the endothelium in cardiovascular pathophysiology. Nat Clin Pract Cardiovasc Med. 2009;6:16-26.

89. Bernard SC, Simpson N, Join-Lambert O, Federici C, LaranChich MP, Maïssa N, et al. Pathogenic Neisseria meningitidis utilizes CD147 for vascular colonization. Nat Med. 2014;20:725-31.

90. Join-Lambert O, Lecuyer H, Miller F, Lelievre L, Jamet A, Furio L, et al. Meningococcal interaction to microvasculature triggers the tissular lesions of purpura fulminans. J Infect Dis. 2013;208:1590-7.

91. Melican K, Michea Veloso P, Martin T, Bruneval P, Duménil G. Adhesion of Neisseria meningitidis to dermal vessels leads to local vascular damage and purpura in a humanized mouse model. PLoS Pathog. 2013;9:e1003139. 